\title{
A CHARACTERIZATION OF CERTAIN QUASI-OPEN MAPPINGS
}

\author{
ROBERT H. KASRIEL ${ }^{1}$
}

1. Introduction. In this paper we shall deal with mappings (continuous functions) defined on regions (open and connected) subsets of a plane $P$ with the range of the functions also contained in $P$. In reference [1], M. K. Fort defined such a mapping $f$ to be minimal provided that for each closed 2-cell $N$ contained in the domain of $f, f(N) \subset g(N)$ for every mapping $g$ whose domain contains $N$ and which is such that $f|\operatorname{Fr} N=g| \operatorname{Fr} N$. The following result was obtained in [1].

If $f$ is light, then $f$ is open if and only if $f$ is minimal.

In this paper, we prove the following similar theorem for certain quasi-open mappings.

1.1. TheOREM. Let $f: X \rightarrow P$ be a compact mapping defined on a simply connected region $X$ in a plane $P$ with $f(X) \subset P$. Then $f$ is quasi-open if and only if $f$ is minimal and for no $x \in f(X)$ is it true that $f^{-1}(x)$ separates $X$.

The sufficiency of the condition in 1.1 is established in $\$ 2$ and the necessity in $\$ 3$.

Recall that a mapping $f: X \rightarrow P$ is compact provided that for each compact set $K \subset f(X), f^{-1}(K)$ is compact. $f$ is quasi-open provided that for any $y \in f(X)$ and any open set $U$ in $X$ containing a compact component of $f^{-1}(y), y \in$ int $f(U)$. Here and throughout the paper such terms as interior, closed, closure (cl), boundary (Fr) will always be relative to the containing plane.

With $f: X \rightarrow P$ a mapping into a plane $P$, if $R$ is an elementary region (a bounded plane region whose boundary consists of a finite number of simple closed curves) whose closure is in the domain of $f$ and $x \in P-f(\operatorname{Fr} R)$, then there is defined the topological index or winding number $\mu(x, f, R)$. Use will be made of the following well-known properties of the index.

1.2. $\mu(x, f, R)$ is constant. on every component of $P-f(\operatorname{Fr} R)$.

1.3. Suppose $x \in P-f(\operatorname{Fr} R)$. If $g: X \rightarrow P$ is such that $g(\bar{x})=f(\bar{x})$ for $\bar{x} \in \operatorname{Fr}(R)$, then $\mu(x, f, R)=\mu(x, g, R)$.

1.4. Let $R_{j} ; j=1,2, \cdots, k$ be a sequence of pair-wise disjoint ele-

Received by the editors August 11, 1961.

1 This research supported in part by the National Science Foundation Grant G-7343 at the Georgia Institute of Technology. 
mentary regions in $R$ such that

$$
(\mathrm{cl} R) \cdot f^{-1}(x) \subset \bigcup_{j} R_{j}
$$

Then $\mu(x, f, R)=\sum_{j} \mu\left(x, f, R_{j}\right)$. (See Theorem 3 on p. 126 in [7].)

1.5. If $\mu(x, f, R) \neq 0$, then $x \in f(R)$.

1.6. If $N$ is a closed 2-cell and $f$ is a homeomorphism on $N$, then $\mu(x, f$, int $N)= \pm 1$ for each $x \in f($ int $N)$.

In terms of this index, Fort [1] proved the following.

1.7. With $f: X \rightarrow P$ as before, $f$ is minimal if and only if for each closed 2-cell $N, f(N)=f(\operatorname{Fr} N)+\{p \mid \mu(p, f$, int $N) \neq 0\}$.

2. Compact plane mappings. In this section we make the following assumption.

2.1. $f: X \rightarrow P$ is a compact mapping from a simply connected region in a plane $P$ into $P$.

From a result of Whyburn ( $\$ 10$ in [4]), there exists a factorization $f=l m$ in which $m$ is monotone and compact and $l$ is light and compact. Throughout the middle space $m(X)$ will be designated as $M$.

If the further assumption is made that for no $x \in f(X)$ it is true that $f^{-1}(x)$ separates $X$, it can be shown that no component of $f^{-1}(x)$ separates $X$. But then for no $z \in M$, is it true that $m^{-1}(z)$ separates $X$. 2.3 below then follows from the following result of Whyburn. (See Note 1, p. 313 in [5].)

If $m$ is a monotone mapping from a plane $X$ onto a space $Y$, then $Y$ is a plane if and only if $m$ is compact and $m^{-1}(y)$ does not separate $X$ for each $y \in Y$.

2.3. Let $f$ be as in 2.1 and let $f=\operatorname{lm}$ be a factorization as before. In addition assume that for $x \in f(X), f^{-1}(x)$ does not separate $X$. Then the middle space $M$ is a topological plane.

2.4. Let $f: X \rightarrow P, l, m, M$ be as in 2.3. Then if $N$ is a closed 2-cell such that $N \subset M, m^{-1}$ (int $N$ ) is a simply connected region.

Proof. $M$-int $N$ is connected. Therefore, since $m$ is compact and monotone, $m^{-1}(M-$ int $N)=X-m^{-1}$ (int $\left.N\right)$ is connected. (See 8.3 in [4].) Since $m$ is compact, $m^{-1}(N)$ and thus $\mathrm{cl}\left(m^{-1}\right.$ (int $\left.N\right)$ ) is compact. Thus $m^{-1}$ (int $N$ ) is a plane region whose closure is compact and whose complement in the plane is connected. Hence $m^{-1}$ (int $N$ ) is simply connected.

2.5. Let $f: X \rightarrow P$ be a compact mapping. Let $x \in f(X)$ and let $U$ be an open set such that $f^{-1}(x) \subset U$. Then there exists an open set $V \subset P$ such that $x \in V$ and $f^{-1}(V) \subset U$.

This follows easily from the compactness of $f$.

2.6. Let $f: X \rightarrow P$ be a mapping as in 2.3. Furthermore assume that $f$ 
is minimal. Then $f$ is quasi-open.

ProOF. Let $f=\operatorname{lm}$ be a factorization as in 2.3. By 2.3 the middle space $M$ may be taken to be a complex plane. Let $x \in f(X)$ and suppose $K$ is a component of $f^{-1}(x)$. Let $U$ be an open set in $X$ such that $K \subset U$. From the definition of quasi-open, the proof will be complete if we show that $x$ is an interior point of $f(U)$. Toward this end, let $p=m(K)$. Note that $p \in l^{-1}(x)$. Since $l^{-1}(x)$ is totally disconnected it can be shown that there exists arbitrarily small 2-cells $N$ such that $($ Fr $N) \cdot l^{-1}(x)=\varnothing$ and $p \in$ int $N$. By $2.5, N$ may be chosen so that $m^{-1}(N) \subset U$. Let $G \equiv m^{-1}$ (int $N$ ). By $2.4, G$ is simply connected. Since $f$ is compact, $f^{-1}(x) \cdot \operatorname{cl} G$ is compact and since $f^{-1}(x) \cdot \operatorname{Fr} G=\varnothing$ it follows that $f^{-1}(x) \cdot G$ is compact. Then since $G$ is simply connected, there exists a closed 2-cell $T$ such that $f^{-1}(x) \cdot G \subset$ int $T \subset T \subset G$. Further by $1.7 \mu(x, f$, int $T) \neq 0$. But then from 1.2 and $1.5, f(T) \supset Q_{x}$ where $Q_{x}$ is the component of $P-f(\operatorname{Fr} T)$ that contains $x$. Since $Q_{x}$ is open and $x \in Q_{x} \subset f(T) \subset f(U)$ it follows that $x$ is an interior point of $f(U)$.

3. Compact quasi-open mappings. In this section we make the following assumption.

3.1. $f: X \rightarrow P$ is a compact and quasi-open mapping defined on a simply connected region $X$ in the plane $P$ and $f(X) \subset P$.

Assuming 3.1, it is known (10.2 and 10.4 in [4]) that there is a monotone-light open factorization $f=l m$ for which $m$ is a compact monotone mapping and $l$ is compact, light, and open relative to $f(X)$. Further from the definition of quasi-open as given in the introduction, $f(X)$ is open in $P$ and hence $l$ is open relative to $P$. Furthermore, we have the following.

3.2. For no point $x \in f(X)$ is it true that $f^{-1}(x)$ separates $X$.

Proof. Suppose $f^{-1}(x)$ separates $X$. Then there exists a separation

$$
X-f^{-1}(x)=A+B .
$$

Since $f^{-1}(x)$ is compact $A+f^{-1}(x)$ or $B+f^{-1}(x)$ is compact. Assume that $A+f^{-1}(x)$ is compact. Then $f(A)+x$ is compact. However $A$ is open and is the union of components of point inverses. Hence from the quasi-openness of $f, f(A)$ is open. This is a contradiction since then $x$ would be the boundary of an open set $f(A)$ in the plane.

On the basis of 3.2 and 2.3 with $f$ as in 3.1, the middle space $M$ can be taken to be a plane. Recall that $f(X)$ is open in $P$. Thus there is available the following result of Whyburn.

3.3. (See VIII, 1.1 and 1.11 in [3].) There exists an integer $k$ and $a$ completely scattered set $D \subset f(X)$, such that for each $x \in f(X)-D, l^{-1}(x)$ consists of $k$ distinct points. Furthermore on the set $M-l^{-1}(D), l$ is a 
local homeomorphism. [Note that for each point $x \in D, x$ is an isolated point of $D$. Also $D$ is closed.]

By making use of 3.3 and 2.4, the following can be obtained.

3.4. For each $z \in f(X)-D$, there exists arbitrarily small closed 2-cells $N$ such that $z \in$ int $N \subset N \subset f(X)-D$ and such that $f^{-1}$ (int $N$ ) is the union of a finite number of pairwise disjoint open two-cells $Q_{i}$ for which $f\left(Q_{i}\right)=$ int $N$ and $f^{-1}\left(z^{*}\right) \cdot Q_{i}$ consists of a single component of $f^{-1}\left(z^{*}\right)$ for each $z^{*} \in$ int $N$.

3.5. $X-f^{-1}(D)$ is a connected open set.

Proof. Notice that $X-f^{-1}(D)=m^{-1}\left(M-l^{-1}(D)\right)$ is open and furthermore is connected since $M-l^{-1}(D)$ is connected and $m$ is a compact monotone mapping.

3.6. Definition of $\mu(x)$. On the open connected set $S \equiv X-f^{-1}(D)$ define the function $\mu$ as follows. Let $x \in S$. Then $f^{-1} f(x)$ consists of exactly $k$ compact components. (See 3.3.) Let $K_{x}$ be that component that contains $x$. There exists an elementary region $R$ such that

$$
K_{x} \subset R \subset \operatorname{cl} R \subset X-f^{-1}(D) \text { and }\left(f^{-1} f(x)\right) \cdot \operatorname{cl} R=K_{x} .
$$

It will be shown in 3.7 that $\mu(x, f, R)$ is independent of the particular choice of $R$ provided that $R$ satisfies (1). On that basis the following may be defined:

$$
\mu(x) \equiv \mu(f(x), f, R) .
$$

(Because of the nature of point inverses of $f, \mu$ is a point function version of the function defined in II. 3.4 of [7].)

3.7. $\mu\left(x, f, R_{1}\right)=\mu\left(x, f, R_{2}\right)$ provided that $R_{1}$ and $R_{2}$ satisfy $(1)$.

Proof. Let $Q$ be the component of $R_{1} \cdot R_{2}$ that contains $K_{x}$. There exists an elementary region $R$ such that

$$
K_{x} \subset R \subset \operatorname{cl} R \subset Q \subset R_{1} \cdot R_{2}
$$

From 1.4, $\mu\left(f(x), f, R_{1}\right)=\mu(f(x), f, R)=\mu\left(f(x), f, R_{2}\right)$.

Use will be made of the following in investigating the function $\mu$.

3.8. Young's Modification Theorem. (See [6].) Let $N$ be a closed 2-cell with interior $G$ and boundary $J$. Suppose $X$ is a continuum and $m: X \rightarrow N$ is a monotone mapping of $X$ onto $N$ such that $m^{-1}(G)$ is an open 2-cell. Then there is a mapping $h: X \rightarrow N$ of $X$ onto $N$ such that $h(x)=m(x)$ for $x \in m^{-1}(J)$ and $h^{-1} h(x)=x$ for $x \in m^{-1}(G)$.

3.9. $|\mu(x)|=1$ for each $x \in X-f^{-1}(D)$.

Proof. Let $x \in X-f^{-1}(D)$. Let $R$ be an elementary region admissible for the computation of $\mu(x)$. (See 3.6.) Let $y=m(x) \in M$. Note that $l$ is a local homeomorphism at $y$ and recall that $m$ is compact. 
Hence by 2.5 there exists a closed 2-cell neighborhood $N$ of $y$ such that $m^{-1}(N) \subset R$ and such that $l$ is a homeomorphism on $N$. Let $G=m^{-1}$ (int $N$ ). By $2.4 G$ is an open 2 -cell. Thus 3.8 can be applied to $m \mid m^{-1}(N)$ and using this a modification $h: X \rightarrow M$ of $m$ can be defined such that $h(z)=m(z)$ for $z \in X-G$ and $h$ is one-to-one on $G$. Let $z^{*}$ be the unique point in $G$ such that $h\left(z^{*}\right)=y$. Note also $h^{-1}(y) \cdot R$ $=z^{*}$. Let $W$ be a closed 2 -cell such that $z^{*} \in$ int $W \subset W \subset G$. Note that $l h \mid W$ is a homeomorphism. Then by 1.6 and from the fact that $l h\left(z^{*}\right)=l(y)=f(x)$, it follows that $\mu(f(x), l h$, int $W)= \pm 1$. Further since $\left[(l h)^{-1} f(x)\right] \cdot R=z^{*}$, it follows from 1.4 that $\mu(f(x), l h$, int $W)$ $=\mu(f(x), l h, R)$. Moreover by 1.3 , since $f=l h$ on $\operatorname{Fr}(R), \mu(f(x), f, R)$ $=\mu(f(x), l h, R)$. Combining this with the two previous equalities involving $\mu$ yields $\mu(f(x), f, R)= \pm 1$.

3.10. $\mu$ is continuous on $X-f^{-1}(D)$. (Hence by $3.5 \mu(x)$ is constant on $X-f^{-1}(D)$.)

Proof. Let $x \in X-f^{-1}(D)$ and $K_{x}$ the component of $f^{-1}(x)$ that contains $x$. Choose $R$ as in (1) of 3.6 so that $\mu(x)=\mu(f(x), f, R)$. Choose a closed 2-cell $N$ such that $f(x) \in$ int $N \subset \operatorname{cl} N \subset f(X)-D$ $-f(\operatorname{Fr}(R))$ and is chosen as in 3.4. Let $Q$ be the component of $f^{-1}$ (int $N$ ) that contains $K_{x}$. Since $f\left(Q_{i}\right)=$ int $N$ for each component $Q_{i}$ of $f^{-1}$ (int $N$ ) (see 3.4) and since $f^{-1}(x) \cdot R=K_{x}$, it follows that $Q$ is the only component of $f^{-1}$ (int $N$ ) that intersects $R$.

Now let $y \in Q$. Let $K_{y}$ be the component of $f^{-1}(f(y))$ that contains $y$. However from the way in which $N$ was chosen (see 3.4) $K_{y}$ is the only component of $f^{-1} f(y)$ that intersects $R$. Thus $R$ is admissible for the computation of $\mu(y)$ and $\mu(y)=\mu(f(y), f, R)$. However by 1.2, $\mu(f(y), f, R)=\mu(f(x), f, R)$ and thus $\mu(x)=\mu(y)$.

3.11. Let $f: X \rightarrow P$ be as in 3.1. Then $f$ is minimal.

Proof. Let $N$ be a closed 2-cell in $X$. From 1.5 and 1.7 we need show only that $\mu(x, f$, int $N) \neq 0$ for each $x \in f(N)-f(\operatorname{Fr} N)$. Let $x \in f(N)-f(\operatorname{Fr} N)$. Define $D$ as in 3.3. Let $U_{x}$ be the component of $P-f(\operatorname{Fr} N)$ that contains $x$. There exists a point $y \in U_{x} \cdot[f($ int $N)-D]$. This follows from the quasi-openness of $f$ and the definition of $D$. By $1.2, \mu(y, f$, int $N)=\mu(x, f$, int $N)$. Let $K_{1}, K_{2}, \cdots, K_{h}$ be the components of $f^{-1}(y)$ that intersect and hence are contained in int $N$. There exist elementary regions $R_{1}, R_{2}, \cdots, R_{h}$ such $K_{i} \subset R_{i} \subset \mathrm{cl} R_{i}$ Cint $N$ and $R_{i} \cdot R_{j}=\varnothing$ if $i \neq j$. By $1.4, \mu(y, f$, int $N)=\sum_{i} \mu\left(y, f, R_{i}\right)$. Let $x_{i} \in K_{i}$. Thus, from definition of $\mu, 3.9$, and $3.10\left|\sum_{i} \mu\left(y, f, R_{i}\right)\right|$ $=\left|\sum_{i} \mu\left(x_{i}\right)\right|=h$ and $\mu(x, f$, int $N)=\mu(y, f$, int $N) \neq 0$.

ThEOREM 1.1. The validity of the Theorem 1.1 stated in the introduction now follows from 2.6, 3.2 and 3.11. 


\section{REFERENCES}

1. M. K. Fort, $A$ characterization of plane light open mappings, Proc. Amer. Math. Soc. 2 (1951), 175-177.

2. G. T. Whyburn, Analytic topology, Amer. Math. Soc. Colloq. Publ. Vol. 28, Amer. Math. Soc., New York, 1942.

3. - Topological analysis, Princeton Univ. Press, Princeton, N. J., 1958.

4. - Open mappings on locally compact spaces, Mem. Amer. Math. Soc. No. 1 (1950).

5. - Compactness of certain mappings, Amer. J. Math. 81 (1959), 306-314.

6. J. W. T. Youngs, Homeomorphic approximations to monotone mappings, Duke Math. J. 15 (1948), 87-94.

7. T. Rado and P. V. Reichelderfer, Continuous transformations in analysis, Springer-Verlag, Berlin, 1955.

The Georgia Institute of Technology 